\title{
Effectiveness-NTU Relationships of Parallel-Plate Moving Bed Heat Exchangers
}

\author{
Pedro A. Isaza, Markus Bussmann* \\ Department of Mechanical and Industrial Engineering \\ University of Toronto \\ Toronto, Ontario, Canada, M5G-3S8
}

\begin{abstract}
Moving bed heat exchangers (MBHEs) are used in various industrial processes. Recently, analytical solutions to several MBHE heat transfer problems have been presented in the literature. In this work, the mathematical procedure by which these new solutions are used to construct effectivenessNTU relationships is presented, for parallel-plate configurations. Expressions for both co- and counter-current orientations are outlined. Effectiveness-NTU plots are then generated, and contrasted with those of fluid-fluid systems. As expected, a functionality with respect to the Number-ofTransfer-Units and the Capacity Ratio is observed. A novel dependency with respect to the Biot number is also demonstrated, whereby effectiveness decreases with increasing Biot number, due to the increasing resistance imparted by the diffusion of energy through the solids bulk. These effectiveness-NTU plots can serve as a design platform, which engineers can use to size and rate MBHEs.
\end{abstract}

Keywords-Effectiveness-NTU; Moving Bed Heat Exchangers; Analytical Solutions

\section{INTRODUCTION}

Describing energy transport into and out of beds of granular solids has been an area of active research for a number of years. Recently, research in the field has given increased attention to the mathematical modeling of heat transfer in moving bed heat exchangers. MBHEs typically consist of a bed of particulate solids flowing downwards by gravity, which exchanges thermal energy across a separating wall with a heating or cooling fluid. Compared with alternative technologies like fluidized beds, moving beds offer reduced investment costs, energy consumption and maintenance requirements $[1,2]$. These competitive advantages have prompted their use in processes ranging from nickel production [3, 4] and food sterilization [5], to flue gas abatement and biomass combustion [6, 7]. Most recently, MBHEs have found a use in solar power generation [8].

Moving bed heat exchangers can be organized in various configurations (i.e. parallel-plate, vertical pipe, etc.) and can accommodate different flow orientations (i.e. counter-current, co-current, or cross flow). Recent mathematical investigations into some of these heat transfer problems have identified analytical solutions for both parallel-plate and vertical pipe configurations [9-12]. The results outlined provide a sizing platform that engineers can now use to design and rate the thermal performance of these systems. One step that is absent in this new body of work, is the transformation of these expressions into effectiveness-NTU relationships that are commonly found in the heat exchanger literature.

The purpose of this paper is to detail the mathematical procedure by which effectiveness-NTU relationships can be established for parallel-plate MBHEs, using the recently presented analytical solutions. From the expressions, effectiveness-NTU curves are generated as a function of the dimensionless groups found in the fluid-fluid heat exchanger literature (i.e. Number-of-Transfer-Units and Capacity Ratio), as well as a new dimensionless group (i.e. Biot number).

\section{GOVERNING EQUATIONS}

The experimentally validated assumptions behind the energy model formulated for the parallel-plate MBHE, outlined by Isaza et al. $[9,10]$, include: The system operates under steady-state conditions, and the solids move with constant velocity under local thermal equilibrium [13-17]. The solids enter the exchanger at a constant temperature, and the thermo-physical properties are constant and isotropic. Heat conduction in the solids occurs in the lateral direction only [9] (i.e. negligible axial heat conduction - explored and validated in [12]), while convection occurs in the axial direction. Energy transfer in the cooling/heating fluid takes place via convection only (i.e. a convective coefficient quantifies transport by means of a SiederTate style correlation [18]). An overall heat transfer coefficient $\left(U_{o}\right)$ comprised of resistances in series due to contact (i.e. nearby wall effects) [13-14, 17, 19-20], wall conduction and convection into the heating/cooling fluid describes energy transfer between the domains. Negligible axial heat conduction takes place along the exchanger wall [11]. Viscous energy dissipation, radiation effects, and sources of thermal energy are negligible. Figure 1 presents a schematic of the co- and countercurrent parallel-plate MBHEs under consideration.

Based on the Cartesian geometry in Fig. 1 and the above assumptions, the MBHE problems can be formulated as follows: 


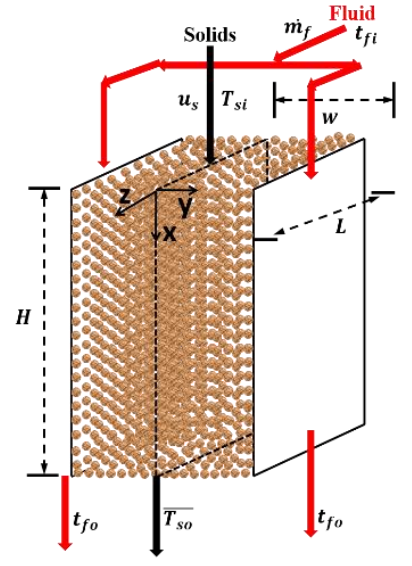

(a)

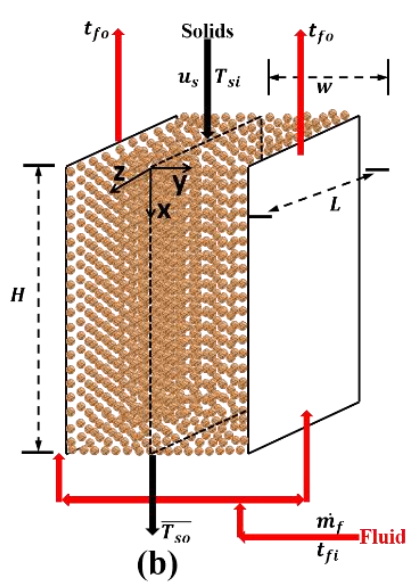

(b)
Figure 1 Co-current (a) and counter-current (b) parallel-plate MBHE schematics

$$
\begin{aligned}
& \rho_{s} C_{p s} u_{s} \frac{\partial T_{S}(x, y)}{\partial x}=k_{s} \frac{\partial^{2} T_{S}(x, y)}{\partial y^{2}} \quad x>0 ; 0<y<w \\
& T_{s}(0, y)=T_{s i} \quad \text { at } \quad x=0 \\
& \left.\frac{\partial T_{S}(x, y)}{\partial y}\right|_{y=0}=0 \quad \text { at } \quad y=0 \\
& -\left.k_{S} \frac{\partial T_{S}(x, y)}{\partial y}\right|_{y=w}=U_{o}\left[T_{s}(x, w)-t_{f}(x)\right] \quad \text { at } \quad y=w
\end{aligned}
$$

where the complementary fluid problem is the following:

$$
\begin{array}{ccc}
U_{o} \cdot L\left[T_{s}(x, w)-t_{f}(x)\right]= \pm \frac{\dot{m}_{f}}{2} \cdot C_{p s} \frac{d t_{f}(x)}{d x} \quad & x>0 \\
t_{f}(0)=t_{f i}(+) & \text { or } \quad t_{f}(H)=t_{f i}(-)
\end{array}
$$

Note that the plus and minus signs correspond to the equations for the co-current [9] and counter-current [10] orientations respectively.

Like other heat transfer investigations, the analytical solutions to the above problems are presented subject to the following nondimensional variables $[9,10]$ :

$$
y^{*}=\frac{y}{w} ; \quad x^{*}=\frac{x}{H} ; \quad \theta_{s}=\frac{T_{s}-t_{f i}}{T_{s i}-t_{f i}} \quad \theta_{f}=\frac{t_{f}-t_{f i}}{T_{s i}-t_{f i}}
$$

Applying these to Eqs. (1) - (6) yields:

$$
\begin{gathered}
\frac{\partial^{2} \theta_{S}\left(x^{*}, y^{*}\right)}{\partial y^{* 2}}=\frac{B i}{N T U} \frac{\partial \theta_{S}}{\partial x^{*}} \\
\theta_{S}\left(0, y^{*}\right)=1 \quad \text { at } x^{*}=0 ; 0<y^{*}<1 \\
\left.\frac{\partial \theta_{S}\left(x^{*}, y^{*}\right)}{\partial y^{*}}\right|_{y^{*}=0}=0 \quad \text { at } \quad y^{*}=0 \\
\left.\frac{\partial \theta_{S}\left(x^{*}, y^{*}\right)}{\partial y}\right|_{y^{*}=1}=B i \cdot\left[\theta_{f}\left(x^{*}\right)-\theta_{S}\left(x^{*}, 1\right)\right] \quad \text { at } y^{*}=1 \\
\frac{d \theta_{f}\left(x^{*}\right)}{d x^{*}}=\mp N T U \cdot C \cdot\left[\theta_{f}\left(x^{*}\right)-\theta_{S}\left(x^{*}, 1\right)\right] \quad x^{*}>0 \\
\theta_{f}(0)=0(-) \quad \text { or } \quad \theta_{f}(1)=0(+)
\end{gathered}
$$

where the minus and plus correspond to the equations for the cocurrent [9] and counter-current [10] orientations. In Eqs. (8) (13) the Biot number $(B i)$, Number-of-Transfer-Units (NTU) and Capacity Ratio $(C)$ dimensionless groups are defined as:

$$
B i=\frac{U_{o} \cdot w}{k_{s}} ; \quad N T U=\frac{U_{o} \cdot H}{\rho_{s} C_{p s} u_{s} w} ; \quad C=\frac{\dot{m}_{s} \cdot C_{p s}}{\dot{m}_{f} \cdot C_{p f}}
$$

\section{ANALYTICAL SOLUTIONS}

Isaza et al. $[9,10]$ present the analytical solutions of the differential equations outlined by Eqs. (8) - (13). The method of solution begins with the application of a Laplace transformation. Once in the Laplace domain, the solid and fluid problems are decoupled, resulting in a well-posed second-order ordinary differential equation problem. Using standard techniques, the problems are solved and the Laplace inverse transform is identified by means of either the standard or the generalized expansion theorem detailed by Luikov [21]. As discussed in [10], to obtain a correct inverse transform for the solids temperature function in the counter-current case, the inverse transform needs to be examined separately for $C<1, C=1$ and $C>1$. This special analysis is driven by the expansion theorem, which requires the identification of the simple roots in the denominator function of the Laplace domain solution. Unlike co-current systems, in the counter-flow problem the roots are a function of $C$, which requires a case-by-case examination. Complete details of the methodology are presented by Isaza et al. [10].

\section{A. Co-Current Analytical Solution}

Following the methodology discussed, Isaza et al. [9] obtained the solution for the co-current problem. As will become evident later, for the purpose of developing effectiveness-NTU relations only the expression quantifying the solids average temperature at the exit is needed. That expression is given as:

$\overline{\theta_{s o}}=\frac{C}{1+C}+\sum_{n=1}^{\infty}\left[\frac{4 \sin \left(\lambda_{n}\right)}{\frac{4 C \sin ^{2}\left(\lambda_{n}\right)}{\lambda_{n}}+2 \lambda_{n}+\sin \left(2 \lambda_{n}\right)}\right] e^{-\frac{\lambda_{n}^{2} \cdot N T U}{B i}} \cdot \frac{\sin \left(\lambda_{n}\right)}{\lambda_{n}}$

which is subject to the transcendental equation:

$\frac{\lambda_{n} \cdot \sin \left(\lambda_{n}\right)}{\left[\frac{C}{\lambda_{n}} \sin \left(\lambda_{n}\right)+\cos \left(\lambda_{n}\right)\right]}=B i \quad$ where $\quad n=1,2,3 \ldots$

\section{B. Counter-Current Analytical Solution:}

For the counter-current configuration, the fluid outlet temperatures in [10] are required to build the effectiveness-NTU relationships. Recall that for counter-flow, the analytical solutions are a function of the capacity ratio, and the case-bycase expressions are presented below:

\section{Fluid Outlet Temperature $-C<1$ :}

$\theta_{f o}=\frac{\frac{C}{1-C}+C \cdot \sum_{n=1}^{\infty}\left[\frac{4 \sin \left(\lambda_{n}\right)}{\frac{4 C \sin ^{2}\left(\lambda_{n}\right)}{\lambda_{n}}-2 \lambda_{n}-\sin \left(2 \lambda_{n}\right)}\right] e^{-\frac{\lambda_{n}^{2} \cdot N T U}{B i}} \cdot \frac{\sin \left(\lambda_{n}\right)}{\lambda_{n}}}{\frac{1}{1-C}+C \cdot \sum_{n=1}^{\infty}\left[\frac{4 \sin \left(\lambda_{n}\right)}{\frac{4 C \sin ^{2}\left(\lambda_{n}\right)}{\lambda_{n}}-2 \lambda_{n}-\sin \left(2 \lambda_{n}\right)}\right] e^{-\frac{\lambda_{n}^{2} \cdot N T U}{B i}} \cdot \frac{\sin \left(\lambda_{n}\right)}{\lambda_{n}}}$ 
Fluid Outlet Temperature $-C=1$ :

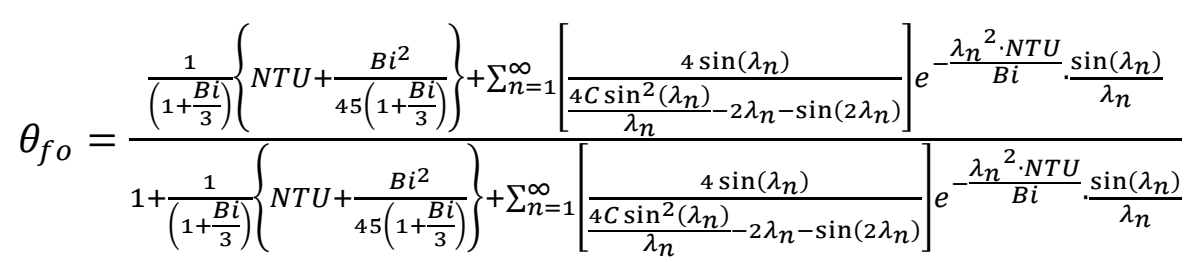

Fluid Outlet Temperature $-C>1$ :

$$
\theta_{f o}=\frac{\frac{C}{1-C}+C\left[\frac{4 \sinh (\mu)}{\frac{4 C \sinh ^{2}(\mu)}{\mu}-2 \mu-\sinh (2 \mu)}\right] \frac{\sinh (\mu)}{\mu} e^{\frac{\mu^{2} \cdot N T U}{B i}}+C \cdot \sum_{n=1}^{\infty}\left[\frac{4 \sin \left(\lambda_{n}\right)}{\frac{4 C \sin ^{2}\left(\lambda_{n}\right)}{\lambda_{n}}-2 \lambda_{n}-\sin \left(2 \lambda_{n}\right)}\right] e^{-\frac{\lambda_{n}^{2} \cdot N T U}{B i} \cdot \frac{\sin \left(\lambda_{n}\right)}{\lambda_{n}}}}{\frac{1}{1-C}+C\left[\frac{4 \sinh (\mu)}{\frac{4 C \sinh ^{2}(\mu)}{\mu}-2 \mu-\sinh (2 \mu)}\right] \frac{\sinh (\mu)}{\mu} e^{\frac{\mu^{2} \cdot N T U}{B i}}+C \cdot \sum_{n=1}^{\infty}\left[\frac{4 \sin \left(\lambda_{n}\right)}{\frac{4 C \sin ^{2}\left(\lambda_{n}\right)}{\lambda_{n}}-2 \lambda_{n}-\sin \left(2 \lambda_{n}\right)}\right] e^{-\frac{\lambda_{n}^{2} \cdot N T U}{B i} \cdot \frac{\sin \left(\lambda_{n}\right)}{\lambda_{n}}}}
$$

subject to the transcendental equations:

$\frac{\lambda_{n} \cdot \sin \left(\lambda_{n}\right)}{\left[\frac{-C}{\lambda_{n}} \sin \left(\lambda_{n}\right)+\cos \left(\lambda_{n}\right)\right]}=B i \quad$ where $\quad n=1,2,3 \ldots$

$\frac{\mu \cdot \sinh (\mu)}{\left[\frac{C}{\mu} \sinh (\mu)-\cosh (\mu)\right]}=B i$

\section{EFFECTIVENESS - NTU RELATIONS}

The effectiveness of a heat exchanger is defined as the ratio of the actual and the maximum heat transfer rate which is achievable in a given system [18, 22]. In the case of the MBHE, the actual heat transfer rate can be defined in terms of the energy lost or gained by the solids or the heating/cooling fluid. The maximum rate of energy transfer on the other hand, is established as the product of the largest temperature differential available in the system and the minimum capacity rate (i.e. the min capacity rate is defined as $c=\dot{m} \cdot C_{p}$ ). Mathematically, the effectiveness of the MBHE can then be defined as:
Equation (22), however, requires further examination as a function of $C$. In cases where $C$ is less than or equal to 1 , the fluid capacity rate exceeds that of the solids (i.e. $\dot{m}_{s} \cdot C_{p s}<$ $\dot{m}_{f} \cdot C_{p f}$ ) and the effectiveness expression adopts the form:

Effectiveness $-C \leq 1$ :

$$
\varepsilon=\frac{\dot{m}_{s} \cdot C_{p s} \cdot\left[\bar{T}_{s o}-T_{s i}\right]}{\dot{m}_{s} \cdot C_{p s} \cdot\left[t_{f i}-T_{s i}\right]}=\frac{\dot{m}_{f} \cdot C_{p f} \cdot\left[t_{f i}-t_{f o}\right]}{\dot{m}_{s} \cdot C_{p s} \cdot\left[t_{f i}-T_{s i}\right]}=1-\overline{\theta_{s o}}=\frac{\theta_{f o}}{C}
$$

Conversely, when $C>1$ the effectiveness expression becomes:

Effectiveness $-C \geq 1$ :

$$
\varepsilon=\frac{\dot{m}_{s} \cdot C_{p s} \cdot\left[\bar{T}_{s o}-T_{s i}\right]}{\dot{m}_{f} \cdot C_{p f} \cdot\left[t_{f i}-T_{s i}\right]}=\frac{\dot{m}_{f} \cdot C_{p f} \cdot\left[t_{f i}-t_{f o}\right]}{\dot{m}_{f} \cdot C_{p f} \cdot\left[t_{f i}-T_{s i}\right]}=\theta_{f o}=C\left[1-\overline{\theta_{s o}}\right]
$$

\section{A. Co-Current Effectiveness Relationships:}

Substituting Eq. (15) into Eqs. (23) and (24) the effectiveness-NTU relations for the co-current MBHE are found to be the following:

$$
\begin{aligned}
& \varepsilon=\frac{1}{1+C}-\sum_{n=1}^{\infty}\left[\frac{4 \sin \left(\lambda_{n}\right)}{\frac{4 C \sin ^{2}\left(\lambda_{n}\right)}{\lambda_{n}}+2 \lambda_{n}+\sin \left(2 \lambda_{n}\right)}\right] e^{-\frac{\lambda_{n}^{2} \cdot N T U}{B i} \cdot \frac{\sin \left(\lambda_{n}\right)}{\lambda_{n}} \quad \text { for } C \leq 1} \\
& \varepsilon=\frac{C}{1+C}-C \sum_{n=1}^{\infty}\left[\frac{4 \sin \left(\lambda_{n}\right)}{\frac{4 C \sin ^{2}\left(\lambda_{n}\right)}{\lambda_{n}}+2 \lambda_{n}+\sin \left(2 \lambda_{n}\right)}\right] e^{-\frac{\lambda_{n}^{2} \cdot N T U}{B i}} \cdot \frac{\sin \left(\lambda_{n}\right)}{\lambda_{n}} \text { for } C \geq 1
\end{aligned}
$$

$$
\varepsilon=\frac{\left[\dot{m} \cdot C_{p} \cdot \Delta T\right]_{s \text { or } f}}{\left[\dot{m} \cdot C_{p}\right]_{\min } \cdot \Delta T_{\max }}
$$

and $\left[\dot{m} \cdot C_{p}\right]_{\min }$ is associated with either the solids or the fluid depending on the conditions examined. Note that the definition applies regardless of the flow configuration.
Note that for the special case where $C=1$, Eq. (26) simplifies to Eq. (25) as expected.

\section{B. Counter-Current Effectiveness Relationships:}

Substituting Eqs. (17) - (19) into Eqs. (23) and (24), the effectiveness-NTU relations for a counter-current MBHE are found to be: 


$$
\begin{aligned}
& \left.\mathcal{E}=\frac{\frac{1}{1-C}+\sum_{n=1}^{\infty}\left[\frac{4 \sin \left(\lambda_{n}\right)}{\frac{1}{1-C}+C \cdot \sum_{n=1}^{\infty}\left[\frac{\sin ^{2}\left(\lambda_{n}\right)}{\lambda_{n}}-2 \lambda_{n}-\sin \left(2 \lambda_{n}\right)\right.}\right] e^{-\frac{\lambda_{n}^{2} \cdot N T U}{B i} \cdot \frac{\sin \left(\lambda_{n}\right)}{\lambda_{n}}}}{\frac{4 C \sin ^{2}\left(\lambda_{n}\right)}{\lambda_{n}}-2 \lambda_{n}-\sin \left(2 \lambda_{n}\right)}\right] e^{-\frac{\lambda_{n}^{2} \cdot N T U}{B i} \cdot \frac{\sin \left(\lambda_{n}\right)}{\lambda_{n}}} \quad C<1
\end{aligned}
$$

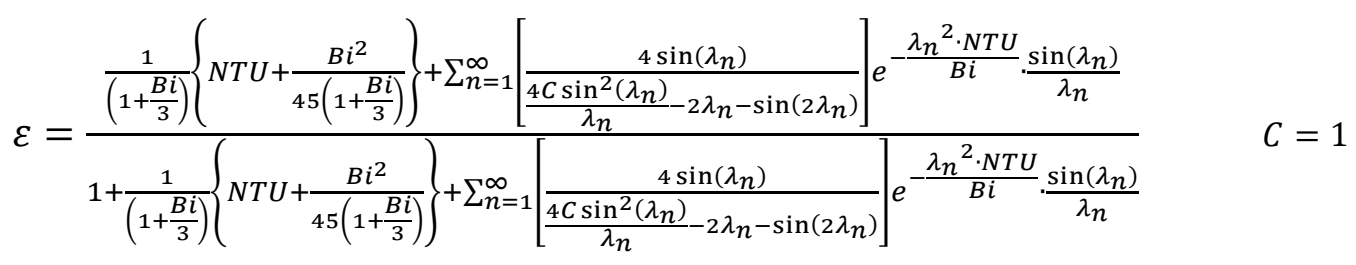

$$
\varepsilon=\frac{\frac{C}{1-C}+C\left[\frac{4 \sinh (\mu)}{\frac{4 C \sinh ^{2}(\mu)}{\mu}-2 \mu-\sinh (2 \mu)}\right] \frac{\sinh (\mu)}{\mu} e^{\frac{\mu^{2} \cdot N T U}{B i}}+C \cdot \sum_{n=1}^{\infty}\left[\frac{4 \sin \left(\lambda_{n}\right)}{\frac{4 C \sin ^{2}\left(\lambda_{n}\right)}{\lambda_{n}}-2 \lambda_{n}-\sin \left(2 \lambda_{n}\right)}\right] e^{-\frac{\lambda_{n}^{2} \cdot N T U}{B i} \cdot \frac{\sin \left(\lambda_{n}\right)}{\lambda_{n}}}}{\frac{1}{1-C}+C\left[\frac{4 \sinh (\mu)}{\frac{4 C \sinh ^{2}(\mu)}{\mu}-2 \mu-\sinh (2 \mu)}\right] \frac{\sinh (\mu)}{\mu} e^{\frac{\mu^{2} \cdot N T U}{B i}}+C \cdot \sum_{n=1}^{\infty}\left[\frac{4 \sin \left(\lambda_{n}\right)}{\frac{4 C \sin ^{2}\left(\lambda_{n}\right)}{\lambda_{n}}-2 \lambda_{n}-\sin \left(2 \lambda_{n}\right)}\right] e^{-\frac{\lambda_{n}^{2} \cdot N T U}{B i} \cdot \frac{\sin \left(\lambda_{n}\right)}{\lambda_{n}}} C 1} \quad C
$$

Several important observations can be made of Eqs. (25) (29). Like fluid-fluid systems [18, 22], the effectiveness of the MBHE is also a function of the Number-of-Transfer-Units $(N T U)$ and the Capacity Ratio $(C)$. This dependency exists regardless of the flow configuration. A new functionality of the MBHE effectiveness with respect to the Biot number is clear from Eqs. (25) - (29). As defined, the Biot number quantifies the magnitude of the resistance associated with lateral energy transfer through the particulate solids. In what follows, the effectiveness dependency on these dimensionless groups is explored graphically.

\section{EFFECTIVENESS - NTU GRAPHICAL ANALYSIS}

For ease of heat exchanger design, it is common for engineers to use the graphical representation of the effectiveness-NTU relations. This is particularly useful during rating exercises, where the area of the exchanger is known and the outlet temperatures need to be determined. As explained by Serth and Lestina [18], this calculation requires an iterative procedure which can be circumvented through the use of effectiveness-NTU plots. This ease of calculation is even more important for MBHEs, due to the presence of the infinite series in the above relations. For all of the plots presented below, the functions are evaluated with 600 terms. This provides sufficient accuracy for the range of dimensionless groups explored [9].

\section{A. Co-Current MBHEs: Effectiveness vs. NTU}

In fluid-fluid heat transfer, it is well known that the effectiveness depends only on $C$ and NTU [18, 22, 23]. Equations (25) and (26), however, clearly demonstrate that for MBHEs $\varepsilon$ also depends on $B i$. Figure 2 presents effectivenessNTU curves for the co-current configuration, as a function of $C$ and $B i$. For comparison purposes, the predictions of the fluidfluid expressions available in the literature are included in the plots.
From Fig. (2a) - (2c), like the fluid-fluid expressions, as the capacity ratio increases (i.e. from 0 to 1 ) the $\mathrm{MBHE}$ effectiveness decreases. This trend exists for all of the Biot numbers explored. Per the definition given by Eq. (22), an effectiveness of one describes a system where the maximum heat transfer rate is achieved. For a co-current system, this occurs when $C=0$, and outlines conditions where the heating/cooling fluid undergoes phase change (i.e. $\dot{m}_{f} \cdot C_{p f}$ tends to infinity). Similarly, when $C=0.5$ and $C=1$ (i.e. Figs. (2b) and (2c)) a maximum effectiveness of 0.67 and 0.5 is obtained. These values agree with the theoretical limits available under cocurrent conditions, whereby the effectiveness tends to $\frac{1}{1+C}$ as $N T U$ goes to infinity (i.e obtained by evaluating the fluid-fluid expressions and Eq. (25) as NTU $\rightarrow \infty$ ). At first glance, Figure (2d) would appear to deviate from this tendency; however, recall from Eqs. (24) and (26) that the effectiveness definition for the MBHE changes when $C$ is greater than one. Unlike fluid-fluid problems, the definition of $C$ here is static (i.e. $C$ is defined as the capacity rate of the solids divided by that of the fluid for all problems), rather than as the ratio of the minimum and maximum capacity rates. This requires the correction of multiplying by $C$, which exists between Eqs. (23) and (24). Having made this correction, Eq. (26) tends to the expected fluid-fluid effectiveness maxima of 0.6 for $C=1.5$. Furthermore, it can be readily shown that as $C$ increases beyond 1.5 , the effectiveness will again tend towards one.

Figure 2 also demonstrates the effect of the Biot number. Figures. (2a) - (2d) show that as the Biot number increases, from 0 (i.e. a "fluid -fluid" analog) to 100 , the $N T U$ required to reach the maximum effectiveness increases. In other words, as the Biot number increases, the area of heat transfer required to reach a given effectiveness also increases. This behavior is independent of the magnitude of $C$, and aligns with the physics of the problem considered. A large Biot number corresponds to a system where the diffusional resistance to lateral heat transfer within the solids is controlling. Under such conditions, the energy exchange 
(a)
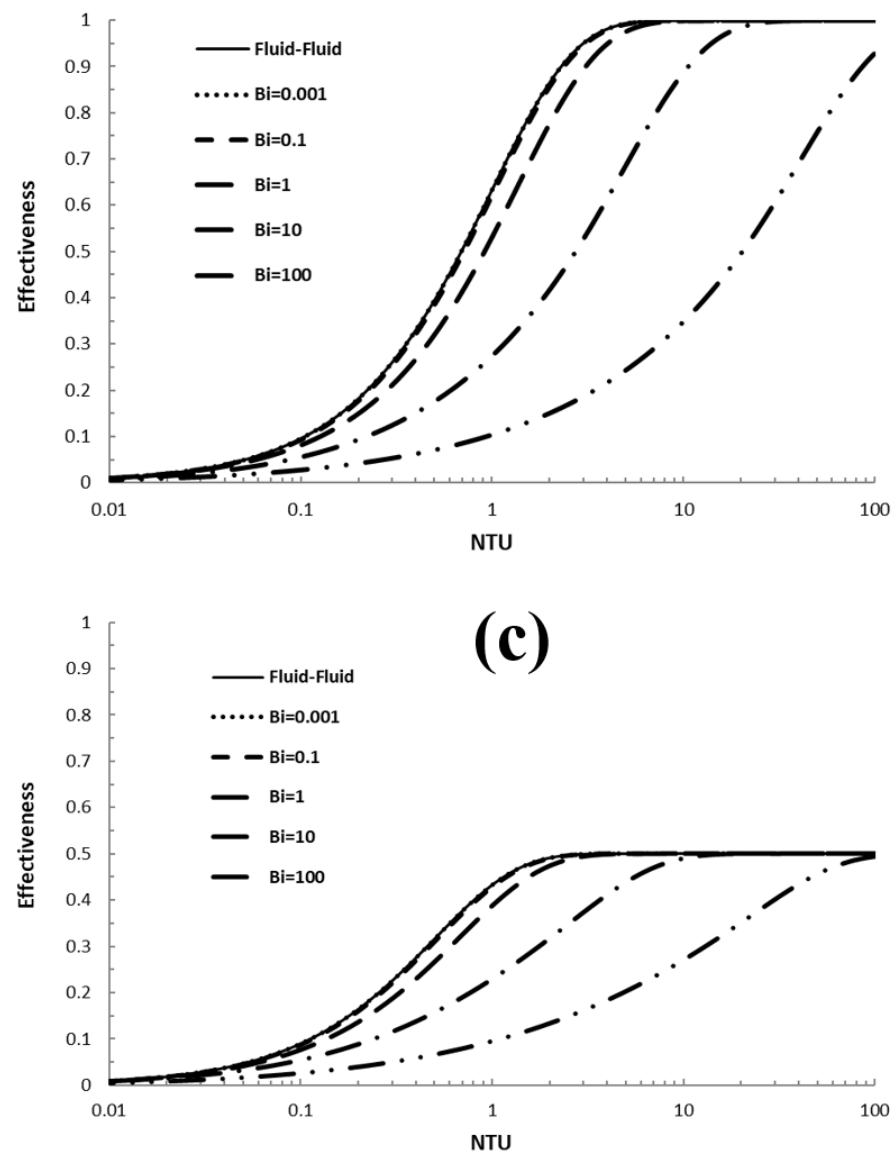

(b)
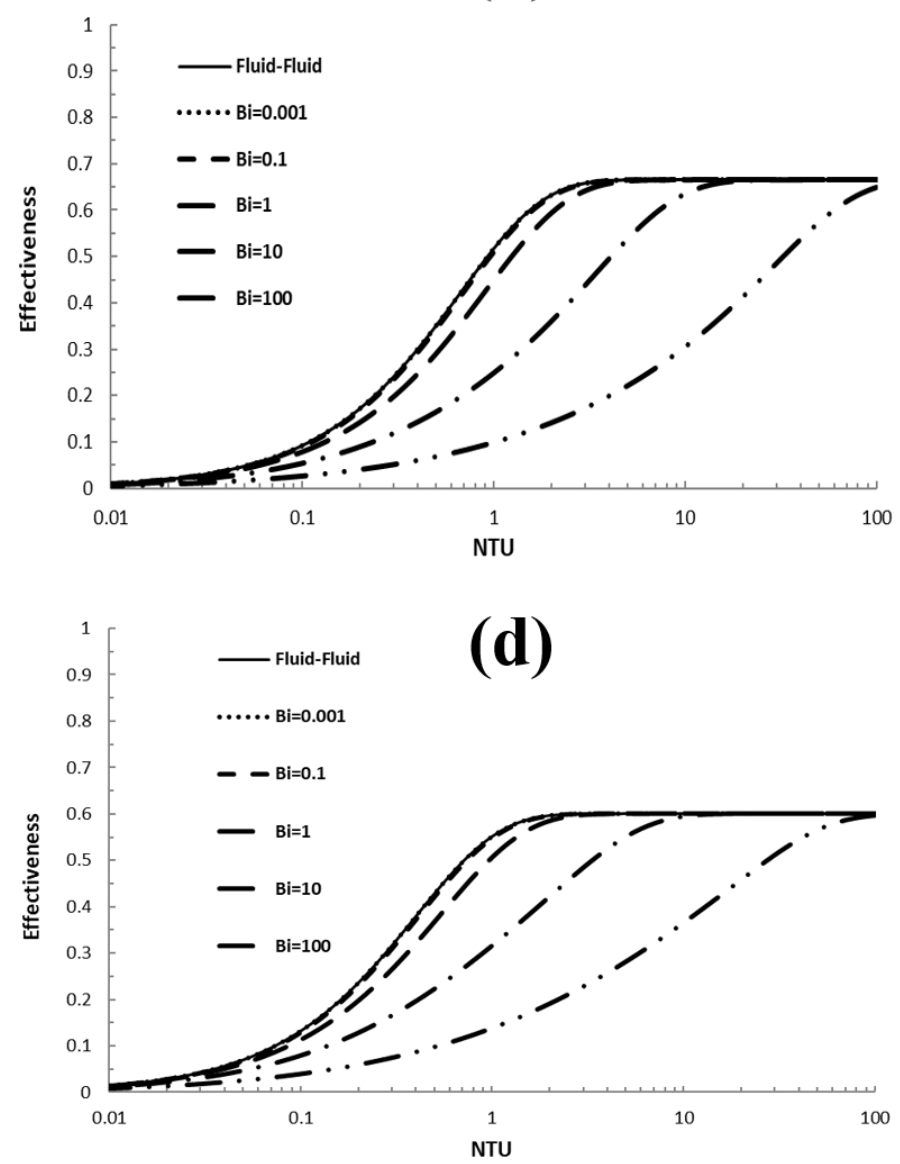

Figure 2. Effectiveness-NTU curves for co-current MBHEs with capacity ratios of (a) $C=0$, (b) $C=0.5$, (c) $C=1$, and (d) $C=1.5$

between the two domains is no longer controlled by the overall heat transfer coefficient (i.e. $U_{o}$ ) and heat transport is controlled by diffusion through the solids bulk. This behavior has been explained in detail by Isaza et al. [9].

\section{B. Counter-Current MBHEs: Effectiveness vs. NTU}

Now we consider the effectiveness-NTU behavior of the counter-current MBHE. As discussed in the literature, counterflow configurations can achieve higher heat transfer rates than co-current systems [24, 25]. As such, for a given amount of energy a smaller area is required. As discussed above, the effectiveness of counter-current fluid-fluid systems depends only on $C$ and $N T U$ [18, 22, 23]. Equations. (27) - (29), however, also demonstrate a Biot number dependency for counter-current MBHEs. Figure 3 presents the effectiveness curves for the counter-current configuration as a function of $N T U, C$ and $B i$. For comparative purposes, the predictions of the fluid-fluid expressions in the literature are included.

The results presented in Fig. 3, display some of the wellestablished characteristics of fluid-fluid systems. First, for all $C$ (i.e. Figs (3a) - (3d)) the effectiveness tends to one. This behavior arises from thermodynamic considerations. In a counter-flow system, given a sufficiently large heat transfer area (i.e. a sufficiently large $N T U$ ), the outlet temperature of the solids will tend to the fluid inlet temperature when $C<1$. From Eq. (23), if $\bar{T}_{s o} \sim t_{f i}$ the effectiveness of the unit tends to one, as per Figs. (3a) - (3c). A similar analysis when $C>1$ also demonstrates that given a sufficiently large area, the fluid outlet temperature will tend to the solids inlet temperature. From Eq. (24), one arrives at the expected maximum effectiveness of 1 observed in Fig. (3d). Secondly, for Figs. (3a) - (3c), as the capacity ratio increases, the $N T U$ required to achieve a given effectiveness also increases. This pattern is independent of the Biot number. For instance, for a Biot number of 1 , the NTU required to reach an effectiveness of 0.95 is $4.05,6.31$ and 25.35 for $C$ equal to $0,0.5$ and 1 respectively.

Figure 3 also depicts some of the Biot number dependencies discussed for the co-current system. For instance, when $C=0$ and $N T U=1$ (i.e. Fig (3a)), the effectiveness are 0.63, 0.62, 0.53, 0.27 and 0.1 for Biot numbers of $0.001,0.1,1,10$ and 100 respectively. Clearly, as the Biot number increases, the effectiveness decreases even under counter-current conditions. 
(a)
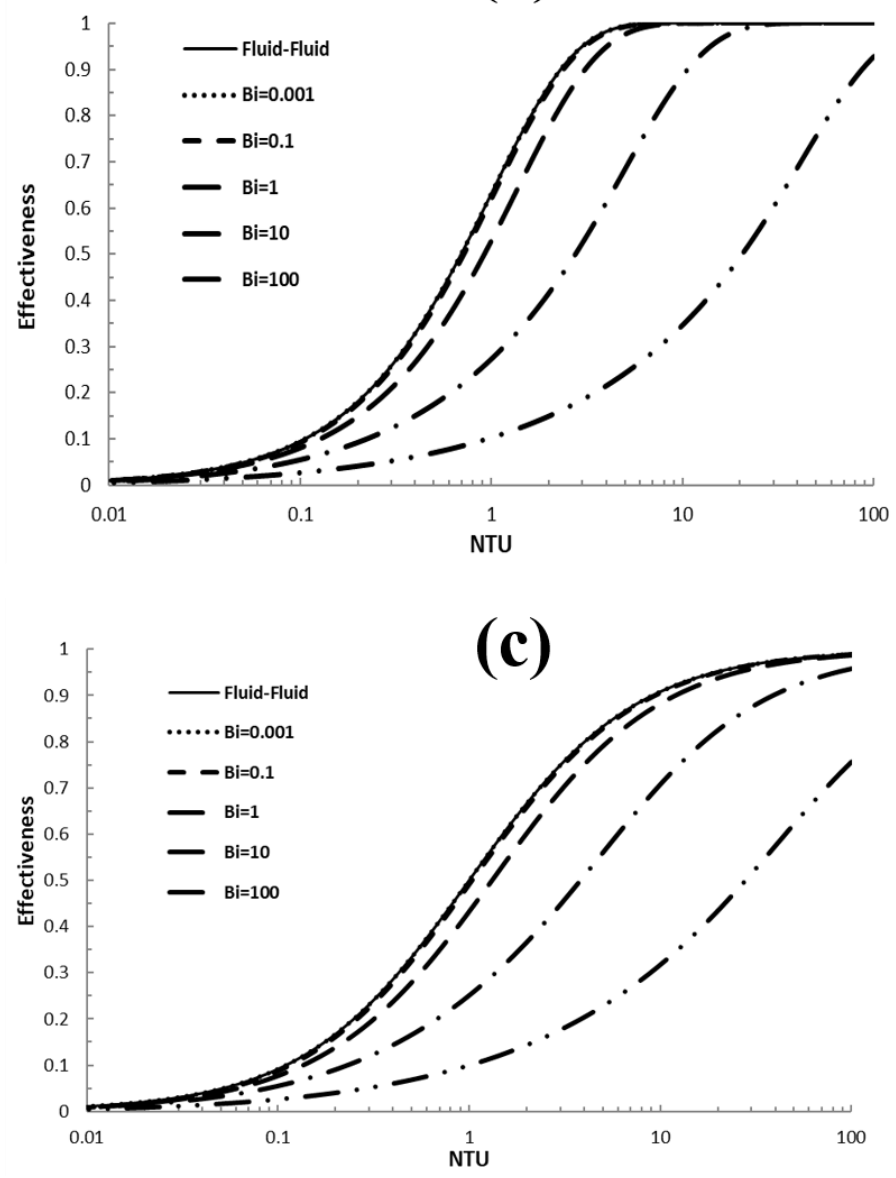

(b)
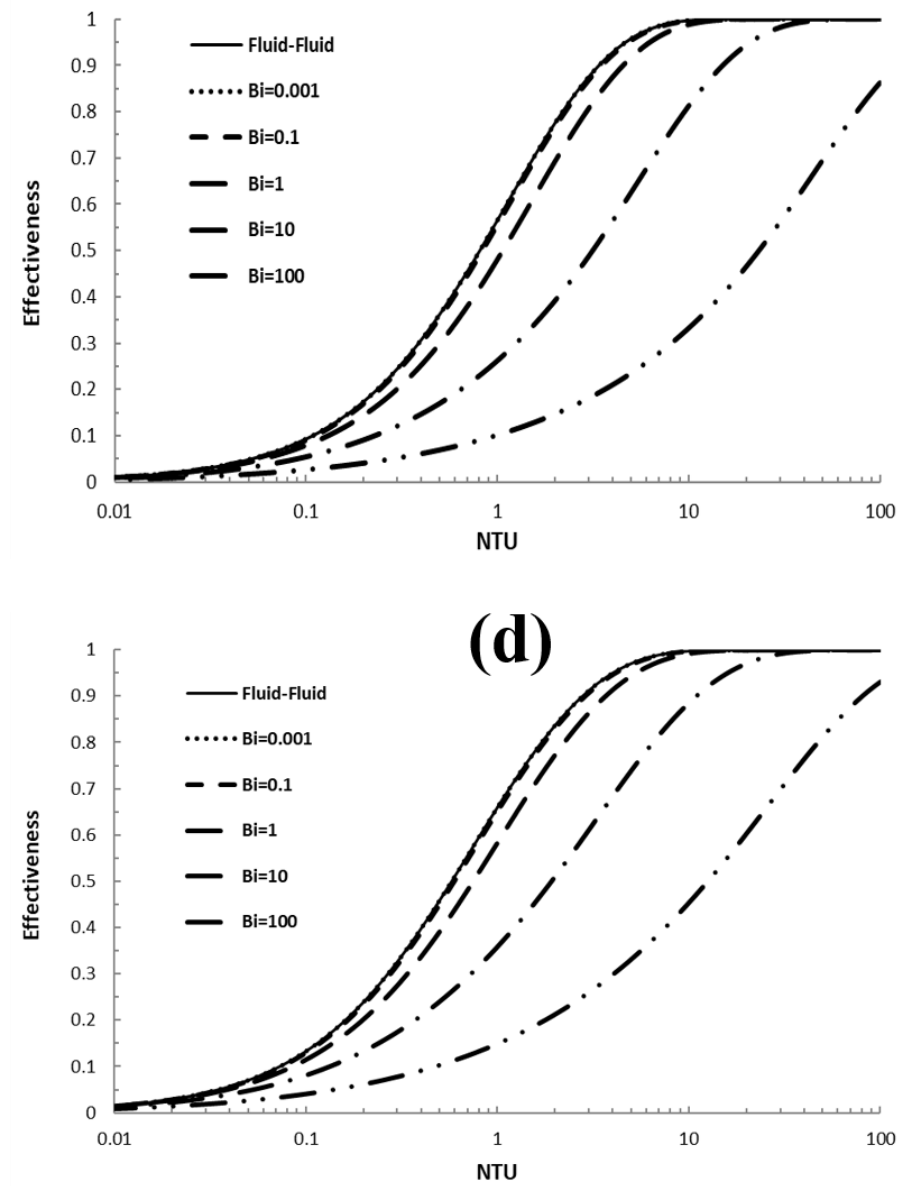

Figure 3. Effectiveness-NTU curves for counter-current MBHEs with capacity ratios of (a) $C=0,(\mathrm{~b}) C=0.5$, (c) $C=1$, and (d) $C=1.5$

By examining Figs. (3a) - (3d), it can be concluded that this dependency exists for all capacity ratios. In other words, regardless of the magnitude of $C$ the effectiveness of the MBHE will decrease with increasing Biot number. As discussed in Section V, large Biot numbers represent a system with a significant diffusional resistance in the solids. This results in the reduced effectiveness that's observed

\section{CONCLUSIONS}

This work presents a methodology for obtaining effectiveness-NTU relationships for parallel-plate MBHEs, based on new analytical solutions found in the literature. Relationships for both co- and counter-current systems are presented. Like fluid-fluid systems, the effectiveness-NTU expressions are found to be a function of the Number-ofTransfer-Units and the Capacity Ratio. Both orientations are shown to be in alignment with the thermodynamic expectations of the systems, and with the corresponding results for fluid-fluid exchangers. A novel dependency of the effectiveness with respect to the Biot number is observed. In particular, as the Biot number increases the effectiveness of the MBHE decreases. This is the case for both co-current and counter-current orientations. A large Biot number is associated with an increased diffusional resistance through the solids bulk, explaining the reduced effectiveness. The relationships and curves presented in this work can serve as a sizing and rating platform for design activities.

\section{NOMENCLATURE}

$\begin{array}{ll}B i & \text { Biot number, }=\frac{U_{o} \cdot w}{k_{s}} \\ C & \text { Capacity ratio, }=\frac{\dot{m}_{s} \cdot C_{p s}}{\dot{m}_{f} \cdot C_{p f}} \\ C_{p f} & \text { Fluid specific heat capacity } \\ C_{p s} & \text { Solids specific heat capacity } \\ H & \text { Plate height } \\ k_{S} & \text { Solids "effective" thermal conductivity } \\ L & \text { Plate depth }\end{array}$




\begin{tabular}{|c|c|}
\hline$\dot{m}_{f}$ & Fluid mass flow rate \\
\hline$\dot{m}_{s}$ & Solids mass flow rate \\
\hline$n$ & Integer number, positive \\
\hline$N T U$ & Number of transfer units, $=\frac{U_{o} \cdot A_{h x}}{\dot{m}_{s} \cdot C_{p s}}=\frac{U_{o} \cdot H}{\rho_{s} u_{s} w C_{p s}}$ \\
\hline$T_{s}$ & Solids temperature \\
\hline$T_{s i}$ & Solids entrance temperature \\
\hline$\overline{T_{s o}}$ & Solids average outlet temperature \\
\hline$t_{f}$ & Fluid temperature \\
\hline$t_{f i}$ & Fluid entrance temperature \\
\hline$t_{f o}$ & Fluid outlet temperature \\
\hline$U_{o}$ & Overall heat transfer coefficient \\
\hline$u_{s}$ & Solids velocity \\
\hline$w$ & Plate half width \\
\hline$x$ & Axial spatial coordinate \\
\hline$x^{*}$ & Dimensionless axial spatial coordinate, $=\frac{x}{H}$ \\
\hline$y$ & Lateral spatial coordinate \\
\hline \multirow[t]{2}{*}{$y^{*}$} & Dimensionless lateral spatial coordinate, $=\frac{y}{w}$ \\
\hline & Greek Letters \\
\hline$\varepsilon$ & Heat exchanger effectiveness, $=\frac{\left[\dot{m} \cdot C_{p} \cdot \Delta T\right]_{s o r f}}{\left[\dot{m} \cdot C_{p}\right]_{\min } \cdot \Delta T_{\max }}$ \\
\hline$\theta_{f}$ & Dimensionless fluid temperature function, $=\frac{t_{f}-t_{f i}}{T_{s i}-t_{f i}}$ \\
\hline$\theta_{f o}$ & Dimensionless fluid outlet temperature, $=\frac{t_{f o}-t_{f i}}{T_{s i}-t_{f i}}$ \\
\hline$\theta_{s}$ & Dimensionless solids temperature function, $=\frac{T_{s}-t_{f i}}{T_{s i}-t_{f i}}$ \\
\hline$\overline{\theta_{s o}}$ & Dimensionless solids average exit temperature \\
\hline$\lambda_{n}$ & $\mathrm{n}^{\text {th }}$ eigenvalue \\
\hline$\rho_{f}$ & Fluid density \\
\hline$\rho_{s}$ & Solids "effective" density \\
\hline
\end{tabular}

\section{REFERENCES}

[1] M. Colakyan, Moving Bed Heat Transfer and Fluidized Elutriation. PhD thesis, Oregon State University, Oregon, USA, 1985.

[2] A. C. Pivem and M. J. S. de Lemos, "Laminar heat transfer in a moving porous bed reactor simulated with a macroscopic two-energy equation model," International Journal of Heat Mass Transfer, vol. 55, no. 5, pp. 1922-1930, 2012.

[3] M. H. I. Baird, N. V. Rama Rao, E. Tackie, and A. Vahed, "Heat transfer to a moving packed bed of nickel pellets," The Canadian Journal of Chemical Engineering, vol. 86, no. 2, pp. 142-150, 2008.
[4] R. Henda and D. J. Falcioni, "Modeling of heat transfer in a moving packed bed: Case of the preheater in nickel carbonyl process," Journal of Applied Mechanics, vol. 73, no. 1, pp. 47-53, 2006.

[5] J. J. Saastamoinen, "Heat exchange between two coupled moving beds by fuid flow," International Journal of Heat and Mass Transfer, vol. 47, no. 6, pp. 1535-1547, 2004.

[6] H. W. Pennline and J. S. Hoffmann, "Flue gas cleanup using the moving bed copper oxide process," Fuel Processing Technology, vol. 114, pp. 109117, 2013.

[7] J. Yrjola and J. J. Saastamoinen, "Modelling and practical operation results of a dryer for wood chips", Drying Technology, vol. 20, no. 6, pp. 10771099, 2002.

[8] T. Baumann and S. Zunft, "Theoretical and experimental investigation of a moving bed heat exchanger for solar central receiver power plants," Journal of Physics: Conference Series, vol. 395, pp. 1-9, 2012.

[9] P. A. Isaza, W. D. Warnica, and M. Bussmann. "Co-current parallel-plate moving bed heat exchanger: An analytical solution," International Journal of Heat and Mass Transfer, vol. 87, pp. 616-624, 2015.

[10] P. A. Isaza, W. D. Warnica, and M. Bussmann. "Counter-current parallelplate moving bed heat exchanger: An analytical solution," International Journal of Heat and Mass Transfer, vol. 87, pp. 625-635, 2015.

[11] P. A. Isaza, Y. Cai, W. D. Warnica, and M. Bussmann. "Co-current and counter-current vertical pipe moving bed heat exchangers: Analytical solutions," International Journal of Heat and Mass Transfer, vol. 95, pp. 1115-1128, 2015.

[12] P. A. Isaza, A. O'Brien, W. D. Warnica, and M. Bussmann. "Assessing axial heat conduction in moving bed heat exchangers," International Journal of Thermal Sciences, vol. 120, pp. 303-313, 2017.

[13] M. Colakyan and O. Levenspiel, "Heat transfer between moving bed of solids and immersed cylinders," AIChE Symposium Series, vol. 80, pp. 241, Union Carbide Corp., Engineering and Technology Services, Research and Development Department, Technical Center, South Charleston, WV, 1984.

[14] W. N Sullivan and R. H. Sabersky, "Heat transfer to flowing granular media," International Journal of Heat and Mass Transfer, vol. 18, no. 1, pp. 97-107, 1975.

[15] M. S. Brinn, S. J. Friedmen, F. A. Gluckert, and R. L. Pigford, "Heat transfer to granular materials," Industrial and Engineering Chemistry, vol. 40, no. 6, pp. 1050-1061, 1948.

[16] N. K. Harakas and K. O. Beatty, "Moving bed heat transfer: Effect of interstitial gas with fine particles," Chemical Engineering Symposium Series, vol. 59, pp. 1050-1061, 1963.

[17] E. U. Schlunder, "Heat transfer to packed and stirred beds from the surface of immersed," Chemical Engineering and Processing, vol. 18, no. 1, pp. 3153, 1984.

[18] R. W. Serth and T. G. Lestina, Process Heat Transfer: Principles, Applications and Rules of Thumb. New York, USA, Academic Press, 2014.

[19] T. Ohmori, M. Miyahara, M. Okazaki, and R. Toei, "Heat transfer in a conductive heating agitated dryer," Drying Technology, vol. 12, pp. 299328, 1994.

[20] M. Kwapinska, G. Saage, and E. Tsotsas, "Continuous versus discrete modelling of heat transfer to agitated beds," Powder technology, vol. 181, no. 3, pp. 331-342, 2008.

[21] A.V. Luikov, Analytical Heat Diffusion Theory. New York, USA: Academic Press, 1968.

[22] W. M. Kays, and A. L. London, Compact Heat Exchangers. New York, USA: McGraw-Hill, Inc., 1984.

[23] J. .P. Holman, Heat Transfer, New York, USA: McGraw-Hill, Inc., 1990

[24] A. C. Pivem and M. J. S. de Lemos, "Temperature distribution in a counterflow moving bed under a thermal nonequilibirum condition," Numerical Heat Transfer Part A: Applications, vol. 61, no. 1, pp. 1-17, 2012.

[25] S. Kakac, H. Liu, and A. Pramuanjaroenkij, Heat Exchangers: Selection, Rating and Thermal Design. Florida, USA: CRC Press., 2012. 